\title{
A New "Multiplex Content" Displaying System Compatible with Current 3D Projection Technology
}

\author{
Koki NAGANO \\ Tokyo Institute of Technology \\ Akihiko SHIRAI* \\ Kanagawa Institute of Technology \\ CSWC, Tokyo Institute of Technology \\ Takeru UTSUGI \\ Tokyo Institute of Technology \\ Masayuki NAKAJIMA \\ Tokyo Institute of Technology
}
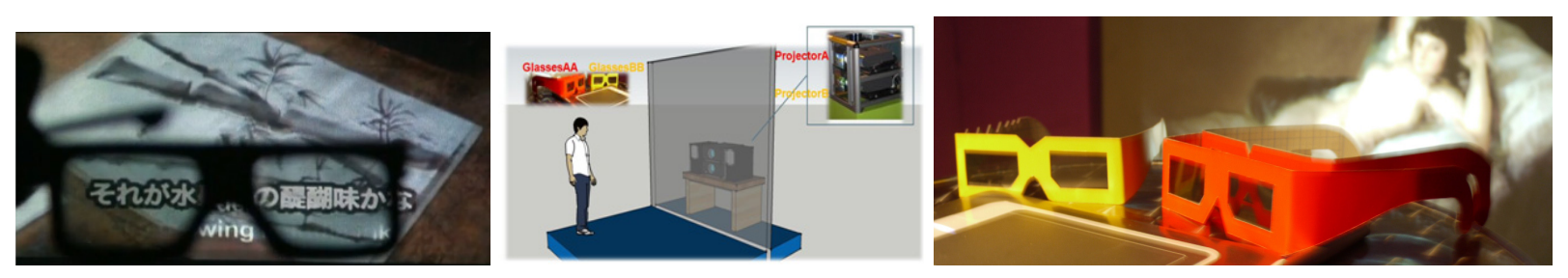

Figure 1: Multiplexed subtitles can be filtered in realtime (left), system configure (middile), contents example: Naked/Clothed Maja(right)

\begin{abstract}
We have enabled the superimposition of multiplexed images on the same screen at the same time with tangible and stable equipment. Our multiplex images can be seen by wearing special configured polarized glasses, and the image projection method is designed to be based on current 3D stereoscopic technology, which is now prevalent and making rapid progress, thus high compatibility with current contents industries is retained. Therefore our system enables the wide range of applications with new expressions and can easily be put into production.
\end{abstract}

\section{Motivation: Giving expansive variations for current 3D viewing technology}

This article suggests a new method for contents creation and broadens horizons of current 3D technologies. Presently, the contents of 3D digital cinemas are rapidly improving as many more theaters start installing 3D digital projectors. These stereo graphics, that are realized with active or passive glasses, enhance the expression of digital content and help prevent illegal copies of cinemas. Consequently, we utilize the infrastructures of 3D graphic projection as a means of contents productions and suggest new contents, "multiplexed contents".

\section{Technology: retained compatibility with current 3D stereo display system}

We have realized the multiplex with no special modification to current major stereoscopic projection methods in order to expand new expressions of contents with the compatibility and sustainable growth. In the passive stereo system, we have configured two different image channels by using polarized DMD projectors (Christie DS+300W / ProjectionDesign F22SX+), instead of stereoscopic images of $\mathrm{L}$ and $\mathrm{R}$, and two pairs of special configured glasses polarized differently between the pairs, but identical between the right and left eye. The synchronized source channels have been generated by network distributed contents players. Especially in the active stereo system, the glasses are electrically-activated time- division. The system is more complicated but it can realize more channels than that of passive by assigning channels to each viewer.

As the passive stereo prototype that is connected with two PCs by our contents player program, we screened two different contents, "The Naked Maja" with Japanese subtitles and "The Clothed Maja" with French subtitles, and prepared two types of glasses so that visitors can enjoy the double contents by changing the glasses. We could confirm that people from wide range of races and cultures can enjoy the different contents without explanation just by selecting the glasses at Laval Virtual ReVolution 2010.

\section{Applications: Tangible Contents Selectors}

Already realized applications are as follows: (1) multi subtitles in cinema; (2)multi cultural commentary for arts in multi subtitles; (3) multi player drawing application. In connection with (2), we enabled not only multi subtitles but also the channels change depending upon user' s age and comprehensive ability. Moreover,we created the magnifier shaped glasses and realized the seamless change of channels. As the specialized feature of this system, we can recognize the value in the idea that users can easily choose information by the glasses, not but by the system. Also other possible applications resonated with people are as follows: (4) multiplex of both video game and visual contents on a domestic screen ; (5) sharing messages with video from SNS like Twitter on public screen ; (6) information display on digital signage depending on types of glasses filter and one' s attributes ; (7) educational contents ; (8) game development ; (9) combination with Augmented Reality techniques. We hope our system will be applied for content platforms like video games and $3 \mathrm{D}$ projectors standards and it will enrich new expressions of digital contents.

\section{References}

SHIRAI, A., ET AL. [information display]. Japan patent application, filed No. 2010-088213 (6th April 2010), (IPC:G06F 21/20).

Takeo Hamada, Koki Nagano, T. U. A. S. Scritter: A multiplexed image system for a public screen. Proceedings of Virtual Reality International Conference (VRIC) Laval Virtual ReVolution 2010 (April 2010), pp. 321-323.

\footnotetext{
*e-mail:scritter@shirai.la,shirai@mail.com
} 